Meta

Journal des traducteurs

Translators' Journal

\title{
Colloque : traduction et qualité de langue
}

\section{J. Bossé-Andrieu}

Volume 28, numéro 2, juin 1983

URI : https://id.erudit.org/iderudit/001890ar

DOI : https://doi.org/10.7202/001890ar

Aller au sommaire du numéro

Éditeur(s)

Les Presses de l'Université de Montréal

ISSN

0026-0452 (imprimé)

1492-1421 (numérique)

Découvrir la revue

Citer cet article

Bossé-Andrieu, J. (1983). Colloque : traduction et qualité de langue. Meta, 28(2), 220-221. https://doi.org/10.7202/001890ar d'utilisation que vous pouvez consulter en ligne.

https://apropos.erudit.org/fr/usagers/politique-dutilisation/ 
suite. La première est que, même si l'histoire prouve que la traduction a eu des effets positifs au cours des siècles, le volume sans cesse croissant de la traduction, au Québec en particulier depuis les années 70 , menace la qualité de la langue française (la notion de qualité de la langue étant d'ailleurs difficile à définir), menace accentuée par le fait qu'un pourcentage élevé de traductions est confié à des pseudo-traducteurs; ensuite, en ce qui a trait à la formation des traducteurs professionnels, il a été souligné qu'il importait que les écoles forment, non pas des traducteurs spécialisés, mais des spécialistes de la traduction, et donc des spécialistes de la langue.

Au cours de la première matinée, consacrée à l'évaluation de la présence de la traduction au Québec et de ses conséquences, J. Poisson et des représentants des médias écrits et parlés ( $P$. Morisset du Devoir et R. Dubuc, de RadioCanada) ainsi que de la publicité (M. Watier) se sont accordés pour dénoncer les méfaits du recours massif à la traduction qu'effectuaient, souvent dans de mauvaises conditions, des gens non initiés, et la contamination langagière engendrée par l'invasion de la culture américaine. Mary Plaice, traductrice du français vers l'anglais, a prouvé que les interférences jouaient dans les deux sens et que les traductions anglaises n'échappaient pas aux gallicismes. Une note d'espoir dans ce sombre tableau: si la traduction est un mal nécessaire, elle peut être un bien puisque, comme l'a démontré P. Claxton, c'est par le biais de la traduction que nous avons accès à des cultures et à des auteurs étrangers.

La séance suivante, intitulée «Traduction et qualité des langues de spécialité ", a vu se succéder plusieurs conférenciers qui ont traité tour à tour des incidences de la traduction sur la terminologie au Québec (L.-J. Rousseau, de l'OLF), de la présence (variable selon le niveau des cours et l'expérience des enseignants) du livre français dans l'enseignement collégial et universitaire du Québec (Y.-A. Côté), des difficultés que présente la traduction des textes de loi (M. Sparer) et de la politique de «traduction tous azimuts» pratiquée par le gouvernement fédéral (J. Gordon). Puis Ch. Faure (Standard Life) a fait le portrait de ce que devrait être le traducteur d'une entreprise en voie de francisation; loin de se cantonner à son rôle de gratte-papier obscur, le traducteur devrait sortir de son bureau pour établir la communication avec les utilisateurs, bref se faire «vendeur » et «diplomate». Enfin, la séance s'est terminée sur l'exposé des avantages et des inconvénients de l'informatisation (A. Guitard: Bell Canada) et sur des réflexions inspirées par le caractère éminemment sociolinguistique de la traduction au Québec (D. Juhel, Université Laval). 
Le dernier jour, les différentes communications ont porté sur les moyens d'atteindre la qualité recherchée. Dans un premier temps, J.-C. Corbeil (CIRELFA), dans son exposé tourné vers le $\mathrm{XXI}^{\mathrm{e}}$ siècle, a prédit un accroissement du volume de traductions, mais, parallèlement, un accroissement du nombre de professionnels dont les tâches seront d'adapter et de rédiger des textes originaux. Ensuite, J.-C. Gémar (Université de Montréal) a brossé un tableau de la formation universitaire idéale du traducteur, tableau complété par une proposition concrète de $G$. Vitale (Université de TroisRivières) qui a esquissé les grandes lignes d'un programme universitaire de traduction. Puis, dans la même veine, $\mathbf{R}$. Roberts (Université d'Ottawa) a décrit les différentes composantes de la «compétence multi-dimensionnelle» que doivent posséder les diplômés en traduction dont la formation n'est complète que s'ils ont effectué un stage professionnel en cours d'études.

Par la suite, G.-M. Boivin (Radio-Canada) a livré quelques réflexions sur la formation des communicateurs non traducteurs - dont la recherche de la pureté de la langue est souvent entravée par des contraintes matérielles avant que ne soient examinés les rapports traducteurs-clients par B. de Vienne et G. Lambert.

Comme on pouvait s'y attendre, la dernière séance a tenté de redéfinir le rôle du traducteur en matière de qualité de la langue. Des exposés des deux traducteurs (J.F. Joly et P. Marchand) et du linguiste (J. Mauras) qui se sont penchés sur la question, se sont dégagés quelques points qui ont semblé rallié l'assistance d'une part, l'obligation, pour le traducteur, d'assumer un double rôle puisque, s'il lui est interdit de nuire à la qualité de la langue, il doit néanmoins contribuer à l'évolution de celle-ci d'autre part, la nécessité d'une revalorisation du métier de traducteur et d'une politique de rapprochement des professions concernées par la qualité de la langue, initiative qui pourrait incomber au Conseil de la langue francaise. Finalement, c'est le président du Conseil de la langue française, $M$. Plourde, qui, par une synthèse magistrale, a clos le colloque.

En conclusion, on peut juger très fructueuses ces deux longues journées au cours desquelles les participants ont entendu les exposés de vingt-cinq professionnels de la traduction et de la communication et suivi les débats sereins et instructifs qui en ont découlé. Plusieurs grandes idées-forces se sont dégagées de ce colloque et ont pris l'allure de recommandations implicites: en premier lieu, bien que, à l'ère des communications, il soit utopique de vouloir supprimer la traduction au Québec, en revanche, il faut, pour réduire le danger d'acculturation, que la création se fasse de plus en plus en français; ensuite, il est essentiel que le traducteur cesse de se considérer - et d'être considéré - comme un simple "gratte-papier transcodeur» et que, conscient de sa responsabilité sociale il «sorte de sa coquille», apprenne à s'imposer auprès de son client et devienne un communicateur; enfin, il est indispensable qu'une formation appropriée, axée sur la connaissance de la langue, prépare le futur traducteur à jouer ce rôle et à exercer un métier par le biais duquel il perpétuera notre patrimoine linguistique et culturel. 